\title{
Los social media y su participación en la construcción y comprensión del relato interactivo de los videojuegos online
}

\author{
Silvia Martínez-Martínez \\ Universitat Oberta de Catalunya \\ smartinezmartinez1@uoc.edu \\ http://orcid.org/0000-0002-6666-7954
}

\author{
Sandra Sanz Martos \\ Universitat Oberta de Catalunya \\ ssanzm@uoc.edu \\ https://orcid.org/0000-0003-3028-852X
}

\section{Social media and its participation in the construction and understanding of the interactive story of online videogames}

\begin{abstract}
RESUMEN
Según Goldstein, Bukingham y Brougère (2004), jugar puede reforzar los vínculos sociales. Si ello lo trasladamos al entorno digital donde el juego es una de las actividades que mayor interés despierta (Sánchez-Navarro, Aranda y Martínez-Martínez, 2015) y le sumamos la posibilidad de intervenir online en videojuegos multijugador, se abre una nueva dimensión en la que los social media pueden

ser una herramienta más que facilite la interacción de los participantes. Los jugadores buscan espacios en los

que compartir informaciones sobre el juego y trucos, también buscar compañeros que les ayuden a superar un reto o incluso brindar interpretaciones y completar así la

narración que ofrece el videojuego. En este sentido los social media pueden servir como aliados para la creación

de comunidades en donde sus miembros, además de interactuar, establecen vínculos de confianza y se identifican en torno a una historia común: el juego. El objetivo de esta comunicación es aproximarse a la presencia y uso de los

social media por parte de jugadores de los principales videojuegos online y analizar las posibilidades que brindan para la construcción y comprensión del relato interactivo de estos en tanto que facilitadores de la creación de comunidades de valor (Sanz-Martos y López-Borrull, 2015).
\end{abstract}

PALABRAS CLAVE

Medios sociales, Videojuegos multijugador online, Relato interactivo, Comunidades de valor

\section{ABSTRACT}

According to Goldstein, Bukingham and Brougère (2004), playing can reinforce social bonds. In digital environment, where the game is an activity that awakens great interest (Sánchez-Navarro, Aranda and Martínez-Martínez, 2015) and where the possibility of online intervention in multiplayer videogames is added, a new dimension is opened in which social media can be one more tool that facilitates the interaction of the participants. The players look for places in which to share information about the game and tricks, also to look for partners who help them overcome a challenge or even provide interpretations and thus complete the story that the video game offers. In this sense, social media can serve as allies for the creation of communities where their members, in addition to interacting, establish bonds of trust and identify themselves around a common history: the game. The objective of this paper is to approach the presence and use of social media by players of the main online videogames. Also it analyzes the possibilities they offer for the construction and understanding of the interactive story of digital games as facilitators of the creation of communities of value (Sanz-Martos and López-Borrull, 2015).

\section{KEYWORDS}

Social media, Multiplayer online game, Interactive story, Communities of value 


\section{Dimensión e impacto del videojuego en el ecosistema mediático}

Los datos de penetración y consumo del videojuego permiten valorar su popularización. Así, según El GAMETRACK Digest de ISFE, para el último cuatrimestre de 2017, el porcentaje de usuarios de algún juego en cualquier formato o plataforma era del 43\% en Gran Bretaña, el 64\% en Francia, el 56\% en Alemania y el $44 \%$ en España. Estos datos son especialmente significativos si se atiende a los patrones de comportamiento de la población joven. Según una encuesta realizada entre personas de edades comprendidas entre los 16 y 35 años en España, el 91\% admite que "ha jugado a videojuegos" (Aranda, 2017). Todo ello lleva a asegurar que "los videojuegos son la primera opción de ocio audiovisual en España" (AEVI, 2017). Asimismo, las cifras que mueve la industria en términos de negocio también contribuyen a corroborar esta realidad. "La facturación de la industria española alcanzó en 2016 los 617 millones de euros" y, según la previsión de crecimiento, "supondría alcanzar en 2020 los 1.440 millones de euros de facturación (DEV, 2017).

Con todo el videojuego se debe ver como un medio más dentro del ecosistema mediático (Aranda y Sánchez-Navarro, 2013) y nos debemos aproximar a él teniendo en cuenta el discurso complejo que conforman sus diferentes facetas donde inciden, entre otros, factores tecnológicos, y económicos pero también la dimensión comunicativa, cultural, artística, lúdica, académica y científica (Aranda y Martínez-Martínez, 2013).

En este sentido se registra un interés creciente entre los investigadores por abordar y analizar el videojuego. Un informe reciente sobre la producción científica relacionada con este objeto (Aranda, Sánchez-Navarro, Martínez-Martínez, 2016) muestra, a partir del registro de las revistas indexadas que en la base de Scopus en el ámbito de las ciencias sociales, el incremento de artículos publicados en los últimos años que bien de forma primaria o secundaria abordan esta temática (véase Gráfico 1).

La práctica ubicuidad del videojuego y su papel dentro de la cultura digital requieren de una alfabetización rigurosa.

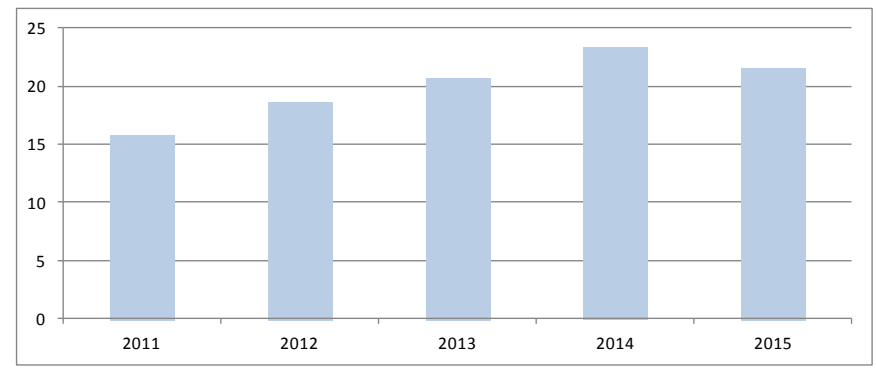

Gráfico 1. Evolución de artículos publicados en revistas del ámbito de las ciencias sociales e indexadas en Scopus que tratan sobre el juego digital. Fuente: Aranda, Sánchez-Navarro, Martínez-Martínez (2016: 25).
En este sentido, no obstante, un informe de 2015 realizado por investigadores de GAME, encargado y financiado por el proyecto European Media Literacy (EMEDUS), en el que se analizan hasta 18 experiencias y proyectos sobre prácticas de alfabetización que contemplan el juego digital, concluye que las "políticas educativas explícitas alrededor del uso del videojuego como objeto de estudio en el contexto de la alfabetización mediática es, dentro del ámbito europeo, casi inexistente" (Aranda, Sánchez-Navarro, Martínez-Martínez, 2015:31). Frente a esta realidad y dada la significación y el rol del videojuego como media en la sociedad y cultura actual, surgen propuestas específicas que hablan de una alfabetización que tenga en cuenta las singularidades del juego digital y que se concreta en la llamada ludoliteracy que, siguiendo a Zagal (2010), que a su vez parte de la propuesta de Gee (2004), incluye capacidades funcionales, es decir, la habilidad para jugar; capacidades analíticas y reflexivas, esto es habilidad para entender los significados y realidades vinculadas al videojuego; y la capacidad productiva que incluye las habilidades necesarias para crearlos.

\section{La percepción del videojuego}

Al margen de estas propuestas e iniciativas, el individuo y la sociedad se construyen una imagen del videojuego. La percepción del juego digital varía e incluye desde aspectos positivos o negativos relacionados con beneficios y perjuicios. Ciertamente la discusión sobre los efectos del juego es una constante que pivota entre voces críticas que apuntan, por ejemplo, a la representación de la violencia o imágenes discriminatorias, el aislamiento y la adición; y otras más favorables que destacan la potencialidad que el juego puede tener en tanto que producto simbólico y social para el aprendizaje (Parra et al. 2009; López-Redondo, 2012:10; Levis, 2005; Aranda y Sánchez-Navarro, 2009).

La percepción se modela y evoluciona. En este sentido, Checa (2009) señala el cambio que, desde una perspectiva cualitativa, ha habido en la imagen que tiene del videojuego la sociedad europea y también la española. A su vez, esta percepción puede variar en función del grupo poblacional de ahí que diferentes estudios se hayan ocupado de analizar la apreciación del juego digital que se registra desde diferentes perfiles y franjas de edad. Así el informe Videogames in Europe: 2012 Consumer Study (Ipsos MediaCT, 2012) recoge la visión de padres con hijos jugadores y señala que el 58\% ven una influencia positiva en el desarrollo de habilidades mientras que el $27 \%$ consideran que impactan en términos de agresividad. La encuesta realizada por investigadores del grupo GAME (Aranda, 2017) en España entre jóvenes de 16 a 35 años concluye que el inconveniente que más señalan es la adición (61\%) mientras que entre los beneficios percibidos con los que se muestran más de acuerdo son la estimulación de la memoria y la capacidad para resolver problemas. Destaca también un 57\% considera que ayudan a estrechar lazos y 
desarrollar nuevas amistades.

En la construcción de la imagen o representación del videojuego inciden múltiples factores que van desde la experiencia personal hasta los mensajes difundidos por los medios de comunicación y los intercambios producidos entre la propia comunidad de jugadores. En este sentido la proliferación en el contexto digital de información y contenidos dedicados a los videojuegos es creciente y supone una competencia a canales y medios convencionales (López-Redondo, 2012). En Internet se multiplican los espacios donde tanto profesionales como aficionados y jugadores comparten sus propias experiencias, habilidades e interpretaciones. (Martínez-Martínez, 2014).

\subsection{La comunidad de jugadores como espacio de construcción e intercambio}

Según Goldstein, Bukingham y Brougère (2004), jugar puede reforzar los vínculos sociales. Calleja (2011), por su parte, propone un modelo de participación que existe entre jugador y juego que comprende tanto el momento de interacción pero también el espacio y el tiempo que van más allá del juego. Estos podrían ser elementos que actúen de motor para la creación de comunidades de jugadores online si se entiende por tal no sólo el entorno virtual de intercambio y comunicación sino también de construcción en el que el factor de cohesión y pertenencia es clave (Rheingold, 1993). Los jugadores buscan espacios en los que compartir informaciones sobre el juego, novedades y trucos, también buscar socios o compañeros que les ayuden a superar un reto o incluso brindar interpretaciones y completar así la historia o la narración que ofrece el videojuego.

El videojuego se presenta como una vía de socialización (Jansz y Marten, 2005). En este sentido el crecimiento de los llamados juegos multijugador online parece facilitar que los usuarios de estos juegos se pongan en contacto. Contreras et al (2014:83), en una obra específica sobre el juego multijugador, se aproxima a ellos desde la perspectiva de la configuración de comunidades de aprendizaje y señala que la participación activa con personas con las que comparten ese "sentimiento de pertenencia" puede favorecerlo ya que "las emociones forman parte del proceso de aprendizaje". Para Wirman (2009:177), algo que singulariza a los juegos multijugador es que "las comunidades entre usuarios/públicos se están creando en el mismo acto de jugar" y señala que la interacción se puede dar también fuera del juego. En este contexto, el multiplayer en entornos online también se ve acompañado por el despegue de los social media que a su vez pueden ser una herramienta más que facilite la interacción de los participantes. En este sentido los social media pueden servir como aliados para la creación de comunidades en donde sus miembros, además de interactuar, establecen vínculos de confianza y se identifican en torno a una historia común: el juego.

\begin{tabular}{|c|c|}
\hline Juego & Perfil en Twitter \\
\hline World of Warcraf & @Warcraft_ES \\
\hline FIFA18 & @EASPORTSEsp \\
\hline Battlefield1 & @battlefield_es \\
\hline
\end{tabular}

Tabla 1. Muestra de estudio. Fuente: Elaboración propia.

\section{Metodologia}

El objetivo de esta comunicación es aproximarse a la presencia y uso de los social media por parte de los jugadores de los videojuegos multijugador online y ver si facilitan la creación de comunidades de valor desde las que contribuir a la comprensión del relato interactivo del juego digital. El estudio se enmarca dentro del proyecto Cultura lúdica, competencia digital y aprendizajes financiado por el Ministerio de Economía, Industria y competitividad (CSO2014-57305-P).

Para cumplir con este objetivo se analizan en concreto las cuentas oficiales en español que en Twitter tienen estos juegos. Dadas las propias características que estos ofrecen los juegos multijugador para la interacción con otros usuarios y también debido a que estos están adquiriendo importancia respecto a la penetración que estaba teniendo el singler player, el análisis se centra en tres juegos que pertenecen a sagas destacadas y con evoluciones diferenciadas en términos del multiplayer online. Asimismo se escogen juegos con modelos de narración y de géneros diferentes que permitan ver singularidades específicas y comportamientos diferenciales de las comunidades que puedan formarse en torno a ellos. Se ven así representados juegos de rol, de deportes y shooter o bélico. Asimismo el entorno o mundo en el que se desarrolla la acción varía desde recreaciones de entornos reales hasta creaciones de mundos y personajes fantásticos e imaginarios (véase la muestra de estudio en la Tabla 1).

El análisis exploratorio realizado de tipo cualitativo pretende observar tanto el tipo de interacción y conversación, sujetos participantes y contenido de los mensajes publicados por los perfiles entre el 15 de enero y el 14 de febrero de 2018. Desde esta perspectiva se analizan las posibilidades que brindan pero también el uso efectivo que aplican para la construcción y comprensión del relato interactivo de los juegos en tanto que los social media puedan actuar como facilitadores de la creación de comunidades de valor, es decir, comunidades de práctica $(C d A)$, comunidades de interés o comunidades de aprendizaje (Sanz-Martos y López-Borrull, 2015). En este sentido, se atenderá a criterios definitorios para distinguir los distintos tipos de comunidades como son el factor de cohesión, liderazgo, tamaño de la comunidad y limitación temporal (Sanz-Martos, 2012:80-81). 


\section{Resultados}

Una primera aproximación a los perfiles de Twitter de los juegos multiplayer mencionados permite observar que se trata de perfiles de amplio recorrido pues datan de 2009 y 2011. Se trata, por tanto, de perfiles longevos en Twitter y ello demuestra la capacidad parar renovar y actualizar temas, en algunos casos de forma coherente al lanzamiento de las nuevas versiones como en este caso las objeto de análisis como por ejemplo FIFA18.

La importancia del vínculo entre usuarios de redes sociales ya fue expresada por Christakis y Fowler (2010). En el caso del social media que ocupa el interés de este análisis, la conexión entre los usuarios y el factor de cohesión se manifiesta a través del sentimiento de pertenencia al mostrarse públicamente como follower del perfil (Martínez-Martínez, 2015). No obstante, la disparidad entre la relación de seguidores y followings de los perfiles estudiados es más que evidente (véase Tabla 2).

Las cuentas siguen a pocos usuarios entre ellos profesionales y/o empresas desarrolladoras /distribuidoras y perfiles que se pueden considerar de influencers entre jugadores conocidos, algún medio o periodista especializado, Youtubers, Twich partners o incluso, como en el caso de FIFA 18, futbolistas de élite. Destaca que a pesar de las bajas cifras de following el propio perfil de Battlefield1 se describe como "Comunidad Oficial de Battlefield en España". Ello pone de manifiesto la importancia y reconocimiento que brindan a los followers y que el perfil se sitúa como espacio y punto de cohesión y contacto entre ellos.

Hay que tener en cuenta que en Twitter la posibilidad de que, a través de un retweet, un mensaje y la conversación generada llegue al timeline de un tercero aumenta las opciones de que usuarios que no se integran en esa lista de followers consulten e incluso participen de alguno de los temas propuestos. Asimismo, la incorporación del perfil a las listas creadas o seguidas por usuarios de Twitter así como la consulta y participación a partir de los mensajes públicos incrementa las posibilidades de alcance de los mensajes pero en estos términos se diluye el factor de cohesión en tanto que no se da esa manifestación pública de pertenencia que se genera al convertirse en follower del perfil.

En cuanto a los contenidos, se observa el interés por las informaciones, novedades y anuncios como por ejemplo los relativos a competiciones y batallas o sobre nuevos mapas y extensiones. Pero también se presenta la oportunidad de compartir consejos, trucos y aclaraciones. En este sentido, por ejemplo, en el perfil de Battlefield1 son llamativos los vídeos de Battle Show donde se ofrecen desde explicaciones de las funcionalidades de un parche hasta descripciones sobre la artillería. Estos vídeos se encuentran publicados en el canal de YouTube BattlefieldEs. En el caso de FIFA18 también algunos de sus tweets remiten a videos, en este caso el espacio El Córner, en su canal oficial de YouTube. En su perfil se observan avisos e informaciones sobre os propios perfiles de los futbolistas que pueden ayudar a los jugadores.

Se observa la creación de hashtags propios como, por ejemplo, \#PorlaAlianza en el caso de World of Warcraf (WoW) y \#FUT o \#TOTW, entre otros, en FIFA18. Estos serán comprendidos en el contexto de juego y por parte de sus usuarios como también ocurra con palabras que se cuelan en los mensajes publicados en los que se encuentran expresiones propias del campo o nombre de batallas como en Battlefield1; que se hable de hordas, taurens o sanadores, en WoW; o que en la FIFA18 se trate sobre cartas o del one to watch. El universo del juego se traslada a los perfiles de Twitter más allá de los términos. Especialmente significativo es el uso de gifs extraídos del mundo fantástico que usa WoW incluso para dar respuesta, sin más referencias, a mensajes de los usuarios. Asimismo publica tweets en los que se citan frases y reflexiones como pronunciadas por los avatares, se indican rasgos característicos de algunos de los personajes y, en algún caso, incluso se usan otros elementos simbólicos, como el color para hablar de la alianza o de la horda. Battlefield1 también publica videos recreando escenas de las batallas mientras que en FIFA18 es más habitual el uso de fotografías en las que se observan los datos clave del perfil de cada futbolista.

En los perfiles se observa la presencia de mensajes más técnicos relativos al juego y su mecánica. Se incluyen aquí los contenidos relativos a parches, extensiones o novedades. También se han podido detectar indicaciones sobre incidencias temporales o avisos para garantizar el adecuado funcionamiento del juego. Se pueden así citar como ejemplos los twwets publicados en el perfil de Battlefield1 donde, el 30 de enero informaba "El multijugador de Battlefield1 está de mantenimiento" o el 25 de enero recordaba el sistema para denunciar a jugadores que infringen "las reglas".

Es significativo que se marquen eventos para encuentros físicos entre los usuarios en los que pueden participar también jugadores profesionales y desarrolladores. Así, en el periodo analizado se observan menciones al EU CUP 1 del Campeo-

\begin{tabular}{|c|c|c|c|c|c|}
\hline \multicolumn{2}{|c|}{ @Warcraft_ES } & \multicolumn{2}{c|}{ @EASPORTSEsp } & \multicolumn{2}{c|}{ Qbbattlefield_es } \\
\hline Following & Followers & Following & Followers & Following & Followers \\
\hline 39 & $74,1 \mathrm{mil}$ & 367 & $418 \mathrm{mil}$ & 89 & $80,8 \mathrm{mil}$ \\
\hline
\end{tabular}

Tabla 2. Datos de followers/followings a partir de la información las cuentas oficiales de los juegos en Twitter con fecha febrero de 2018. Fuente: Elaboración propia. 
nato de Arena en WoW y el FUT Champions Cup en FIFA18. En paralelo también se destacan eventos virtuales que marcan el calendario de los usuarios como el \#FridayNightBattlefield o incluso los viernes de chistes en WoW.

En cuanto a la interacción y conversación mantenidas en estos perfiles, se observa una marcada unidireccionalidad del mensaje derivada del predominio de tweets registrado por parte de la cuenta de Battlefield1 y de FIFA18. En ambos la presencia de respuestas a otros usuarios es prácticamente nula o inexistente. En el caso de FIFA18, se observa mayor número de retweets que en el perfil del juego de batalla e incluyen perfiles oficiales de jugadores de fútbol profesionales, equipos e incluso a la cuenta oficial de la Liga. Ello muestra que la conexión con el mundo real al que simulan también se traslada a este entorno virtual de comunicación.

En WoW el nivel de interacción es mayor siendo especialmente elevado el número de respuestas registrado. Además, se observa un interés por fomentar la participación y relacionarse con los usuarios ya que en sus entradas realiza preguntas, menciona otros perfiles e incluso realiza encuestas. En los tres perfiles es a través de las repuestas de los usuarios a los tweets donde se observa el desarrollo de hilos y conversaciones entre estos al margen de que el perfil oficial no intervenga más allá del mensaje inicialmente publicado. Este nivel de interacción entre usuarios es especialmente significativo en el perfil del juego FIFA18 por la mayor unidireccionalidad que muestra la cuenta oficial y por la constante de conversación/ respuestas que en contrapartida se observa por parte de los usuarios. Se debe señalar, no obstante, que el mayor número de respuestas o intervenciones en el hilo generado que se ha observado procede de un tweet del perfil de WoW del 11 de febrero que conmemoraba la llegada del juego a Europa producido 13 años antes.

\section{Conclusiones}

El estudio permite concluir que se da un comportamiento mixto entre los usuarios de Twitter de los perfiles analizados. Se combina el comportamiento satélite y puntual que imposibilitaría hablar de comunidad (por la falta del factor de cohesión que se observaría entre los lookers o replicantes y retweeteadores esporádicos que, sin embargo, contribuyen a amplificar el alcance del mensaje) con características propias de las comunidades de interés según descripción de Sanz-Martos (2012). Concretamente estos rasgos son los que derivan del factor de cohesión explicitado al convertirse en follower de la cuenta; el volumen de seguidores; el interés común que se renueva con nuevas versiones del juego y nuevos obstáculos o competiciones; la longevidad de dichas comunidades; y la capacidad y facilidad para renovar a sus miembros e incluir nuevos.

El universo del juego se traslada a los perfiles de Twitter tanto por los términos empleados como completando la narración de este y la descripción de los personajes. Además esta recreación o continuación de la ficción, con incluso un lenguaje y uso de imágenes y símbolos propios, fomentarían la cohesión del grupo al generar conversaciones comprendidas únicamente por usuarios/gamers. Lo mismo ocurre con la creación de eventos presenciales pero también con el calendario de actividades virtuales que facilitan mantener el contacto y fijar objetivos comunes. La publicación de consejos, recomendaciones y guías proporcionan claves para participar en el juego y comprenderlo. Los avisos de incidencias puntuales, parches e incluso sobre las reglas del juego irrumpen en el relato de ficción y devuelve a la realidad que enmarca el universo recreado a partir del juego digital.

Si bien se observan diferencias en estas cuentas a la hora de fomentar la participación así como también en los contenidos y patrones de comportamiento que parecen acordes con el modelo, género y narración del videojuego, sería necesario un estudio en profundidad que permita establecer una comparación más amplia de perfiles. La flexibilidad que permite Twitter facilita la presencia de observadores que pueden seguir los contenidos e incluso participar puntualmente en la conversación sin desarrollar el sentimiento de pertenencia a la comunidad, lo que hace su análisis especialmente interesante. No obstante, los resultados también animan a continuar con la investigación incluyendo perfiles en otros social media para establecer pautas y modelos, a partir de similitudes y diferencias detectadas, que a su vez sean extrapolables al estudio de las comunidades de valor que se mueven en estos entornos.

\section{NOTA}

El estudio se enmarca dentro del proyecto Cultura lúdica, competencia digital y aprendizajes financiado por el Ministerio de Economía, Industria y competitividad (CS02014-57305-P).

\section{Referencias}

AEVI (2017). 2016 Anuario de la industria del videojuego. Recuperado de http://www.aevi.org.es/web/wp-content/uploads/2017/06/ ANUARIO_AEVI_2016.pdf

DEV (2017). Libro blanco del desarrollo español del videojuego. Recuperado de http://www.dev.org.es/images/stories/docs/libro\%20 blanco\%20dev\%202017.pdf

Aranda, D. (2017) (coord.) Jóvenes y juego digital. Hábitos de uso y percepciones de los jóvenes sobre la imagen de los videojuegos en los medios de comunicación. Informe ejecutivo. Recuperado de http://hdl. handle.net/10609/66246

Aranda, D., y Martínez-Martinez, S. (2013). Ludoliteracy. Media literacy in gaming. En Aranda, D., Creus, A., y Sánchez-Navarro, J. (eds.). Educación, medios digitales y cultura de la participación (47-68). Barcelona: UOC.

Aranda, D., y Sánchez-Navarro-J. (2009) (eds.) Aprovecha el tiempo y juega. Barcelona: UOC. 
Aranda, D., y Sánchez- Navarro, J. (2013). Ludoliteracy: una reflexión. En: Ludoliteracy, creación colectiva y aprendizajes. Actas del II Congreso Internacional de Educación Mediática y Competencia Digital (Barcelona, 14-15 noviembre 2013), 618-626. Recuperado de http:// www.uoc.edu/portal/es/symposia/congreso_ludoliteracy2013/ conclusiones_actas/index.html

Aranda, D., Sánchez-Navarro, J. y Martínez-Martínez, S. (2015) Ludoliteracy. Informe sobre la alfabetización mediática en el juego digital. Experiencias en Europa. Barcelona: UOC. Recuperado de http://hdl. handle.net/10609/40821

Aranda, D., Sánchez-Navarro, J., y Martínez-Martínez, S. (2016) (coords.). El videojuego en el punto de mira.La producción científica sobre el juego digital. Barcelona: UOC. Recuperado de http://hdl. handle.net/10609/57544

Calleja, G. (2011). In-Game. From Immersion to Incorporation, Cambridge: The MIT Press.

Checa, A. (2009). Hacia una industria española del videojuego. Comunicación, 1(7), 177-188.

Christakis, N.A., y Fowler, J.H. (2010). Conectados, El sorprendente poder de las redes sociales y cómo nos afectan. Madrid: Santillana Ediciones.

Contreras, R. S., Eguia, J. L., y Lozano-Muñoz, A. (2014), Juegos multijugador: El poder de las redes en el entrenamiento. Barcelona: UOC.

Gee, J.P. (2004). Lo que nos enseñan los videojuegos sobre el aprendizaje y el alfabetismo. Málaga: Aljibe.

Goldstein, J., Buckingham, D., y Brougère, G. (2004). Toys, Games and Media, London: LEA.

ISFE; Ipsos Connect (2017). GameTrack Digest: Quarter 3 2017. Recuperado de https://www.isfe.eu/sites/isfe.eu/files/gametrack_european_summary_data_2017_q3.pdf

Ipsos Media CT (2012). Videogames in Europe: 2012 Consumer Study. European Summary Report, November 2012. Commissioned by ISEFE. Recuperado de https://www.isfe.eu/sites/isfe.eu/files/attachments/ euro_summary_-_isfe_consumer_study.pdf

Jansz, J. y Marten, L. (2005). Gaming at a LAN event: the social context of playing videogames. New Media \& Society, 7(3), 333-355.

Levis, D. (2005). Videojuegos y alfabetizacióin digital. Aula de Innovación Ediucativa, (147), 48-50.

López-Redondo, I. (2012). El tratamiento del videojuego: de la prensa generalista a las revistas especializadas, análisis comparativo de las ediciones impresas y digitales de 'El País', 'El mundo', 'Público' y '20Minutos'. [Tesis doctoral]. Sevilla: Universidad de Sevilla. Recuperada de http://fondosdigitales.us.es/

Martínez- Martínez, S. (2014). La información sobre videojuegos como ámbito de especialización periodística. Comunicació: Revista de Recerca y d'Anàlisi, 32(2), 99-114.

Martínez-Martínez, S. (2015). La comunidad de los diarios digitales españoles en Twitter: análisis de la distribución y del perfil de la red de contactos. Anàlisi. Quaderns de Comunicació i Cultura, (52), 17-31.

Parra, D., García de Diego-Martínez, A. y Pérez-Martín, J. (2009). Hábitos de uso de los videojuegos en España entre los mayores de 35 años. Revista Latina de Comunicación Social, (64), 694-707.

Rheingold, H. (1993) The virtual community. Homesteading on the electronic frontier. Reading, Mass: Addison-Wesley.

Sanz-Martos, S., y López-Borrull, A. (2015). Revistas científicas como comunidades de valor. CRECS 2015. Recuperado de http://thinkepi. net/notas/crecs-2015/J12_30ssanzm_alopezbo.pdf

Sanz-Martos, S. (2012). Comunidades de práctica. El valor de aprender de los pares, Barcelona: Editorial UOC.

Wirman, H. (2009). Sobre la productividad y los fans de los videojuegos. En: Aranda, D., y Sánchez-Navarro-J. (eds.) Aprovecha el tiempo y juega. Barcelona: UOC.

Zagal, J.P. (2010). Ludoliteracy: Defining, understanding, and supporting games education. Halifax: ETC Press.

\section{CV}

Silvia Martínez-Martínez. Doctora europea en Comunicación, Licenciada en Ciencias de la Información (Periodismo) por la Universidad CEU Cardenal Herrera. Ha sido galardonada con distintos reconocimientos por su trayectoria académica e investigadora. Desde el 2005 su trayectoria profesional se vincula al mundo académico donde ha disfrutado de diversas becas, entre ellas la Beca de Formación de Personal Universitario concedida por el Ministerio de Educación. En ese periodo realiza dos estancias en centros extranjeros: Communication and Media Research Institute (CAMRI) de la University of Westminster y Observatório do Ciberjornalismo (Obciber) del Centro de Estudos das Tecnologias e Ciências da Comunicação (Cetac. media) en la Universidade do Porto. En 2011 se incorpora como docente a la Universitat Oberta de Catalunya donde, además de profesora, ha sido coordinadora de posgrado en los Estudios de Ciencias de la Información y de la Comunicación. Desde 2006 ha participado en diferentes proyectos vinculados al estudio de la participación ciudadana y la comunicación digital. Colabora en el Observatorio de Investigación en Medios Digitales (OIMED) y es miembro del Grupo de investigación en Aprendizajes, Medios de Comunicación y Entretenimiento (GAME), reconocido como grupo consolidado por AGAUR.

Sandra Sanz-Martos. Doctora en Sociedad de la Información y el Conocimiento (2010) por la UOC. Licenciada en Documentación por la Universidad de Granada (1998) y en Filología Hispánica por la Universidad de Barcelona (1995). Desde septiembre de 1999 es profesora titular de los Estudios de Ciencias de la Información y de la Comunicación de la Universitat Oberta de Catalunya. Actualmente dirige la especialización de posgrado: "Comunicación digital para la transformación social". Como investigadora lleva quince años trabajando en torno al aprendizaje colaborativo, comunidades de aprendizaje y de práctica, trabajo colaborativo e intercambio de conocimiento a través de las redes sociales. Es miembro del grupo de investigación GAME (Grupo de investigación en Aprendizajes, Medios y Entretenimiento) reconocido como grupo consolidado por AGAUR, donde participa -entre otros- en el proyecto I+D Cultura lúdica, competencia digital y aprendizajes. También es Miembro del Grupo ThinkEPI y colaboradora habitual del Anuario ThinkEPI. Análisis de tendencias en información y comunicación. 